\title{
Effect of Noise on Excursions To and Back From Infinity
}

\author{
Jeff Moehlis ${ }^{1}$ \\ Program in Applied and Computational Mathematics, Princeton University, \\ Princeton, NJ, 08544-1000
}

PACS: 05.45.+b, 47.20.Ky, 47.52.+j, 47.54.+r

\begin{abstract}
The effect of additive white noise on a model for bursting behavior in large aspectratio binary fluid convection is considered. Such bursts are present in systems with nearly square symmetry and are the result of heteroclinic cycles involving infinite amplitude states created when the square symmetry is broken. A combination of numerical results and analytical arguments show how even a very small amount of noise can have a very large effect on the amplitudes of successive bursts. Large enough noise can also affect the physical manifestations of the bursts. Finally, it is shown that related bursts may occur when white noise is added to the normal form equations for the Hopf bifurcation with exact square symmetry.
\end{abstract}

Key words: Noise, Bursts

\section{Introduction}

In physical systems, noise is unavoidable. Thus, it is important to study the effect of noise on models of physical systems. It has been shown that some models are very sensitive to the presence of noise: for example, the model for the resonant interaction of three wave modes considered in [1] shows that a tiny amount of noise can replace a bifurcation structure involving a period doubling cascade to chaos with a noisy periodic orbit which is attracting over a wide range of parameter values. Noise can have an especially profound effect on models with structurally stable heteroclinic cycles. These cycles involve connections between unstable states, and the connections typically occur in invariant subspaces. Even small amounts of noise can cause the system to

$\overline{1}$ E-mail: jmoehlis@math.princeton.edu 
jump across these subspaces, and this can lead to very different dynamical behavior [2]. If such a heteroclinic cycle also is attracting, the time spent in the neighborhood of the unstable states of the cycle increases without bound with increasing time. This is not observed in physical systems and noise provides an explanation: the heteroclinic cycle becomes a "statistical limit cycle" in which switching between the unstable states of the cycle occurs randomly in time but with a well-defined mean period [3-5].

In $[6,7]$, a model of large aspect-ratio binary fluid convection was studied. The model considers the competition between two nearly degenerate modes of opposite parity. The evolution equations correspond to a Hopf bifurcation with broken $\mathrm{D}_{4}$ symmetry, where the "interchange" symmetry between the two modes is weakly broken because of the large but finite aspect-ratio. For open parameter regimes, periodic or irregular bursting with very large dynamic range can occur close to threshold. The bursts are associated with nonattracting heteroclinic cycles involving solutions "at infinity" [7,8]. These cycles may be structurally stable or unstable. Bursts occur when the system evolves toward an infinite amplitude state along its stable manifold, then gets kicked toward another infinite amplitude state with an unstable manifold which returns the system to finite amplitude. This mechanism provides an explanation for bursts found experimentally for large aspect-ratio binary fluid convection [9], and may also provide a deterministic explanation for the variability of the solar magnetic cycle [10]. A comparison of this to other mechanisms for bursting is given in $[11,12]$. Related bursts can occur due to the resonant temporal forcing of a system undergoing a Hopf bifurcation with $\mathrm{D}_{4}$ symmetry [13].

When noise is added to the model considered in $[6,7]$, there will be a burst with larger amplitude if the noise kicks the system closer to the stable manifold of an infinite amplitude state, and a burst with smaller amplitude if the noise kicks it away. Thus a state which bursts in the absence of noise is still expected to burst in the presence of noise, but the successive burst amplitudes and the time between successive bursts may change substantially. The details of the effect of noise on the bursting behavior are described in this paper. This includes the possibility that noise can affect the physical manifestations of the bursts. Specifically, purely blinking and winking states in which successive bursts occur at opposite sides or the same side of the container, respectively, can be destroyed by sufficiently large noise. Finally, it is shown that related bursts can occur even if the $\mathrm{D}_{4}$ symmetry of the model is not broken, i.e., when noise is added to the normal form equations for the Hopf bifurcation with exact $\mathrm{D}_{4}$ symmetry. 


\section{Evolution equations for the model}

For binary fluid convection, if the separation ratio of the mixture is sufficiently negative then convection arises via a Hopf bifurcation. This is the case for the ${ }^{3} \mathrm{He} /{ }^{4} \mathrm{He}$ mixture used by Sullivan and Ahlers [9] in their experiment carried out in the large aspect-ratio rectangular container

$$
D \equiv\left\{X, Y, Z \mid-\frac{1}{2} \Gamma \leq X \leq \frac{1}{2} \Gamma,-\frac{1}{2} \Gamma_{Y} \leq Y \leq \frac{1}{2} \Gamma_{Y},-\frac{1}{2} \leq Z \leq \frac{1}{2}\right\},
$$

with aspect-ratio $\Gamma=34$, and $\Gamma_{Y}=6.9$. In this experiment, it was observed that immediately above threshold convective heat transport may take place in a sequence of irregular bursts of large dynamic range despite constant heat input. A model for this experiment is considered in [6,7]. The perturbation to the conduction state temperature profile is assumed to take the form

$$
\Theta(X, Y, Z, t)=\epsilon^{1 / 2} \operatorname{Re}\left\{z_{+}(t) f_{+}(X, Y, Z)+z_{-}(t) f_{-}(X, Y, Z)\right\}+\mathcal{O}(\epsilon)
$$

where $\epsilon \ll 1, f_{ \pm}(-X, Y, Z)= \pm f_{ \pm}(X, Y, Z)$. Here $z_{+}$are $z_{-}$are the (complex) amplitudes of the first modes to lose stability which are respectively even and odd under the reflection $X \rightarrow-X$. In [6], evolution equations for $z_{+}$ and $z_{-}$were derived using symmetry arguments, with the resulting equations describing a Hopf bifurcation with broken $\mathrm{D}_{4}$ symmetry. The applicability of this model to the experiment is discussed further in [14]. As shown in [6,7], solutions of the evolution equations include bursts of very large dynamic range similar to those found in the experiments. The mechanism by which these bursts arise is discussed in detail in [8], and is summarized below.

In this paper we generalize the model considered in $[6,7]$ to include additive random forcing terms. Specifically, we consider the formal equations

$$
\begin{aligned}
\frac{d z_{+}}{d t}= & (\lambda+\Delta \lambda+i(\omega+\Delta \omega)) z_{+}+A\left(\left|z_{+}\right|^{2}+\left|z_{-}\right|^{2}\right) z_{+} \\
& +B\left|z_{+}\right|^{2} z_{+}+C \bar{z}_{+} z_{-}^{2}+\eta_{1}(t)+i \eta_{2}(t) \\
\frac{d z_{-}}{d t}= & (\lambda-\Delta \lambda+i(\omega-\Delta \omega)) z_{-}+A\left(\left|z_{+}\right|^{2}+\left|z_{-}\right|^{2}\right) z_{-} \\
& +B\left|z_{-}\right|^{2} z_{-}+C \bar{z}_{-} z_{+}^{2}+\eta_{3}(t)+i \eta_{4}(t)
\end{aligned}
$$

where the $\eta_{i}$ 's represent real, independent Gaussian white noise random processes with the properties

$$
\left\langle\eta_{i}(t)\right\rangle=0, \quad\left\langle\eta_{i}(t) \eta_{j}\left(t^{\prime}\right)\right\rangle=2 \mathcal{D} \delta\left(t-t^{\prime}\right) \delta_{i j}, \quad i, j=1,2,3,4
$$


Here $z_{+}, z_{-}, A, B, C$ are complex, and $\lambda, \Delta \lambda, \omega, \Delta \omega, \mathcal{D}$ are real. The quantity $\Delta \omega$ measures the difference in frequency between the two modes at onset, and $\Delta \lambda$ measures the difference in their linear growth rates. The terms involving $\Delta \lambda$ and $\Delta \omega$ are called forced symmetry-breaking terms because they break the $\mathrm{D}_{4}$ symmetry of the governing equations [7]. The new $\eta_{i}$ terms represent unavoidable random effects in the experiment.

We first summarize results for the case that there is no noise, i.e., when $\mathcal{D}=0$. New coordinates $(\rho, \theta, \phi, \psi)$ are defined according to

$$
z_{+}=\rho^{-1 / 2} \cos (\theta / 2) e^{i(\phi+\psi) / 2}, \quad z_{-}=\rho^{-1 / 2} \sin (\theta / 2) e^{i(-\phi+\psi) / 2},
$$

where without loss of generality $\theta \in[0, \pi], \phi \in[-2 \pi, 2 \pi)$, and $\psi \in[0,4 \pi)$. Also introducing a new time $\tau$ defined by $d \tau / d t=1 / \rho$, equations $(1,2)$ with $\mathcal{D}=0$ take the form

$$
\begin{aligned}
& \frac{d \rho}{d \tau}=-\rho\left[2 A_{R}+B_{R}\left(1+\cos ^{2} \theta\right)+C_{R} \sin ^{2} \theta \cos 2 \phi\right]-2(\lambda+\Delta \lambda \cos \theta) \rho^{2}(4) \\
& \frac{d \theta}{d \tau}=\sin \theta\left[\cos \theta\left(-B_{R}+C_{R} \cos 2 \phi\right)-C_{I} \sin 2 \phi\right]-2 \Delta \lambda \sin \theta \rho \\
& \frac{d \phi}{d \tau}=\cos \theta\left(B_{I}-C_{I} \cos 2 \phi\right)-C_{R} \sin 2 \phi+2 \Delta \omega \rho \\
& \frac{d \psi}{d \tau}=2 A_{I}+B_{I}+C_{I} \cos 2 \phi+C_{R} \sin 2 \phi \cos \theta+2 \omega \rho .
\end{aligned}
$$

Here $A=A_{R}+i A_{I}$, etc. Let

$$
r=\frac{1}{\rho}=\left|z_{+}\right|^{2}+\left|z_{-}\right|^{2}
$$

denote the amplitude of a solution. As discussed in [7,8], equations (4-7) have an invariant subspace $\Sigma$ at $\rho=0$ (corresponding to infinite amplitude states) on which the equations are equivalent to those with $\Delta \lambda=\Delta \omega=0$. Since the variable $\psi$ decouples from the other variables, the dynamics on $\Sigma$ are two-dimensional and hence simple to analyze [15]. Such an analysis (see [8]) allows us to conclude that, for this truncation, infinite amplitude periodic and quasiperiodic solutions exist. Bursts are associated with heteroclinic cycles involving such infinite amplitude states. Reference [8] shows that for such cycles to form, it is necessary to have at least one subcritical and one supercritical solution branch bifurcating from the trivial state with $\Delta \lambda=\Delta \omega=0$; also, the "angular stability" properties of the solutions must be correct. Reference [8] also shows that despite their heteroclinic nature, the duration of the resulting bursts in the original time $t$ is in fact finite, i.e., the excursion to and return from infinity occur in finite time. Of course, the infinite amplitude solutions 
and connections to them are of physical interest only insofar as they are responsible for the presence of nearby solutions which make visits to large but finite amplitude. This type of bursting behavior persists for $\mathcal{D}=0$ even when higher order terms in equations $(1,2)$ are retained [8]. It should be emphasized that the noise is added on the original timescale $t$, not $\tau$ (see equations $(1,2)$ ).

\section{Effect of noise on bursting behavior}

We now describe the effect of noise on the bursting behavior reported in $[6,7]$. Coefficient values which give the appropriate sub- and supercriticality of traveling and standing wave branches in large aspect-ratio binary fluid convection systems are considered [7]:

$$
\begin{gathered}
A=1-1.5 i, \quad B=-2.8+5 i, \quad C=1+i, \\
\omega=1, \quad \Delta \lambda=0.03, \quad \Delta \omega=0.02 .
\end{gathered}
$$

For these coefficient values in the absence of noise, nonattracting, structurally stable heteroclinic cycles connecting infinite amplitude periodic orbits (hereafter, $u_{\infty}$ solutions) and infinite amplitude quasiperiodic orbits (hereafter, $q p_{\infty}$ solutions) exist over a range of $\lambda$ values. These heteroclinic cycles are of Shil'nikov-Hopf type, and there are associated stable solutions which display bursting behavior $[7,8]$.

For definiteness, consider the stable quasiperiodic solution which is present in the absence of noise for $\lambda=0.1$ (see Figure 1(a); in the notation of [8], this is a $w_{e}^{1}$ solution). The trajectory makes successive visits near different but symmetry-related $u_{\infty}$ solutions given by $(\rho, \theta, \phi, \psi)=\left(0, \pi / 2, m \pi,\left(2 A_{I}+\right.\right.$ $\left.\left.B_{I}+C_{I}\right) \tau \bmod 4 \pi\right), m=0, \pm 1, \pm 2$. The $u_{\infty}$ solutions are unstable within $\Sigma$, and the trajectory gets kicked toward a $q p_{\infty}$ solution. The $q p_{\infty}$ solutions are unstable in the $\rho$ direction, so the trajectory returns to smaller amplitude (larger $\rho$ ). Physically, this solution corresponds to a blinking state for the convection system because successive bursts occur at opposite sides of the container [7]. Figure 1(b) shows the corresponding results for $\mathcal{D}=1 \times 10^{-7}$. Clearly, even this very small amount of noise can have a very large effect on the burst amplitudes. These and other numerical results were obtained using a stochastic second-order Runge-Kutta algorithm [16]. Unless otherwise indicated, the time step of integration is $\delta t=1 \times 10^{-4}$.

The results shown in Figure 1(b) may be qualitatively understood by recognizing that there will be a burst with larger amplitude if the noise kicks the trajectory closer to the stable manifold of a $u_{\infty}$ solution, and a burst with smaller amplitude if the noise kicks it away. This is elucidated by ignoring the 

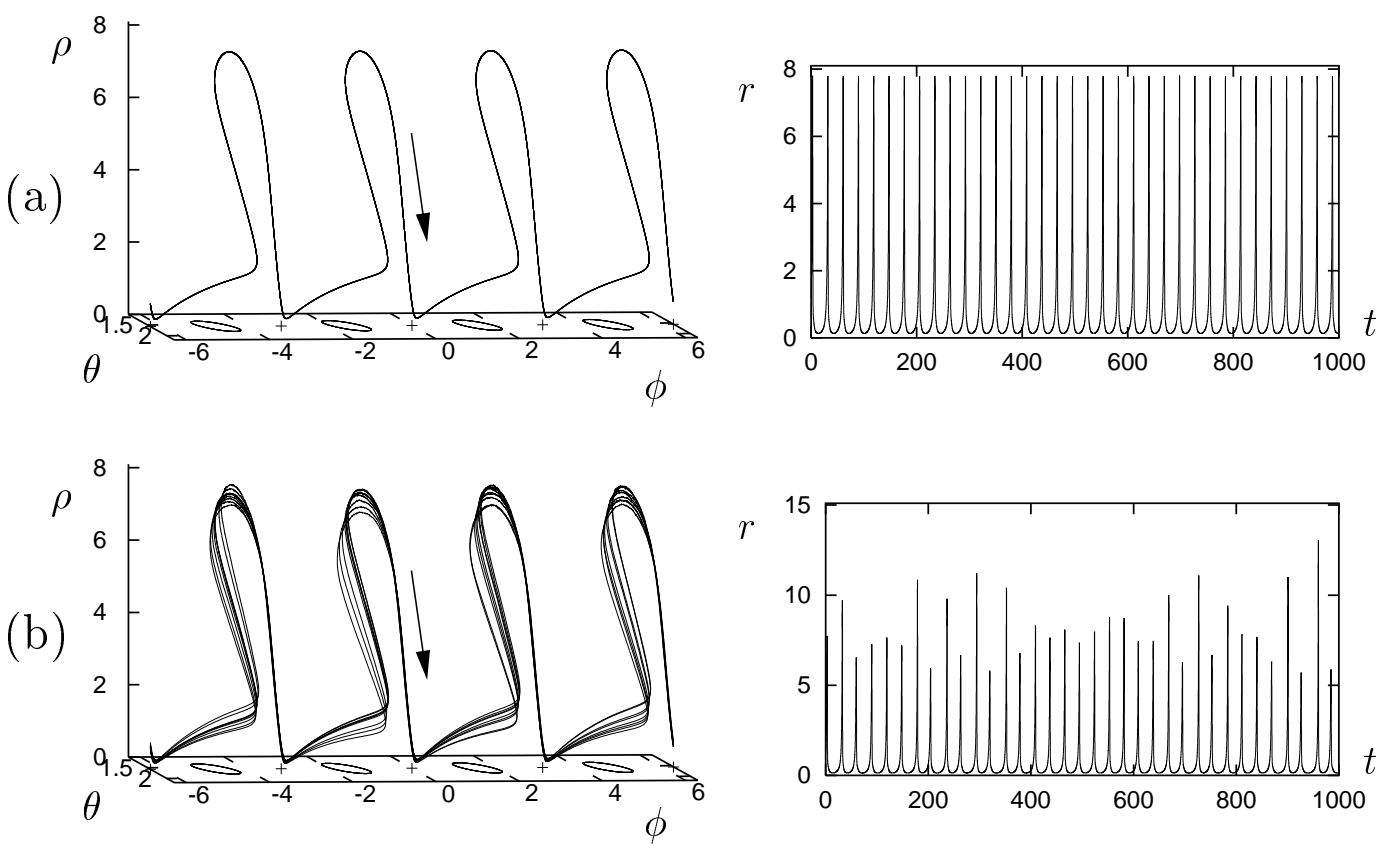

Fig. 1. Bursts for $\lambda=0.1$ and (a) $\mathcal{D}=0$, (b) $\mathcal{D}=1 \times 10^{-7}$. Clearly, even a very small amount of noise can have a very large effect on the burst amplitudes. Fixed points (periodic orbits) in these projections correspond to periodic orbits (quasiperiodic orbits) for the full system. In this and later figures, the $u_{\infty}$ solutions are denoted by + 's, and the $q p_{\infty}$ solutions appear as periodic orbits at $\rho=0$.

uncoupled variable $\psi$, linearizing equations (4-6) about the $u_{\infty}$ solutions, and defining coordinates $(\rho, x, y)$ to give the diagonal form

$$
\frac{d x}{d \tau}=\lambda_{u} x, \quad \frac{d \rho}{\mathrm{d} \tau}=-\lambda_{s} \rho, \quad \frac{d y}{d \tau}=-\lambda_{s s} y
$$

where $\lambda_{u}, \lambda_{s}, \lambda_{s s}>0$. The stable manifold of the $u_{\infty}$ solutions then approximately intersects a plane of sufficiently small, constant $\rho$ along the line $x=0$. For the parameters under consideration, $\lambda_{u}=0.0682, \lambda_{s}=0.2$, and $\lambda_{s s}=5.868$, and equation (8) is obtained by using the coordinates approximately given by

$$
\left(\begin{array}{l}
\rho \\
x \\
y
\end{array}\right)=\left(\begin{array}{ccc}
1 & 0 & 0 \\
0.269 & -0.730 & 0.706 \\
0.00626 & 0.902 & 0.467
\end{array}\right)\left(\begin{array}{c}
\rho \\
\theta-\pi / 2 \\
\phi-m \pi
\end{array}\right) .
$$

Figure 2(a) shows values at which the trajectory intersects the surface $\rho=1$ with decreasing $\rho$ for $\mathcal{D}=1 \times 10^{-7}$. This is interpreted as showing how the noise affects the proximity of the trajectory to the stable manifold of the $u_{\infty}$ 

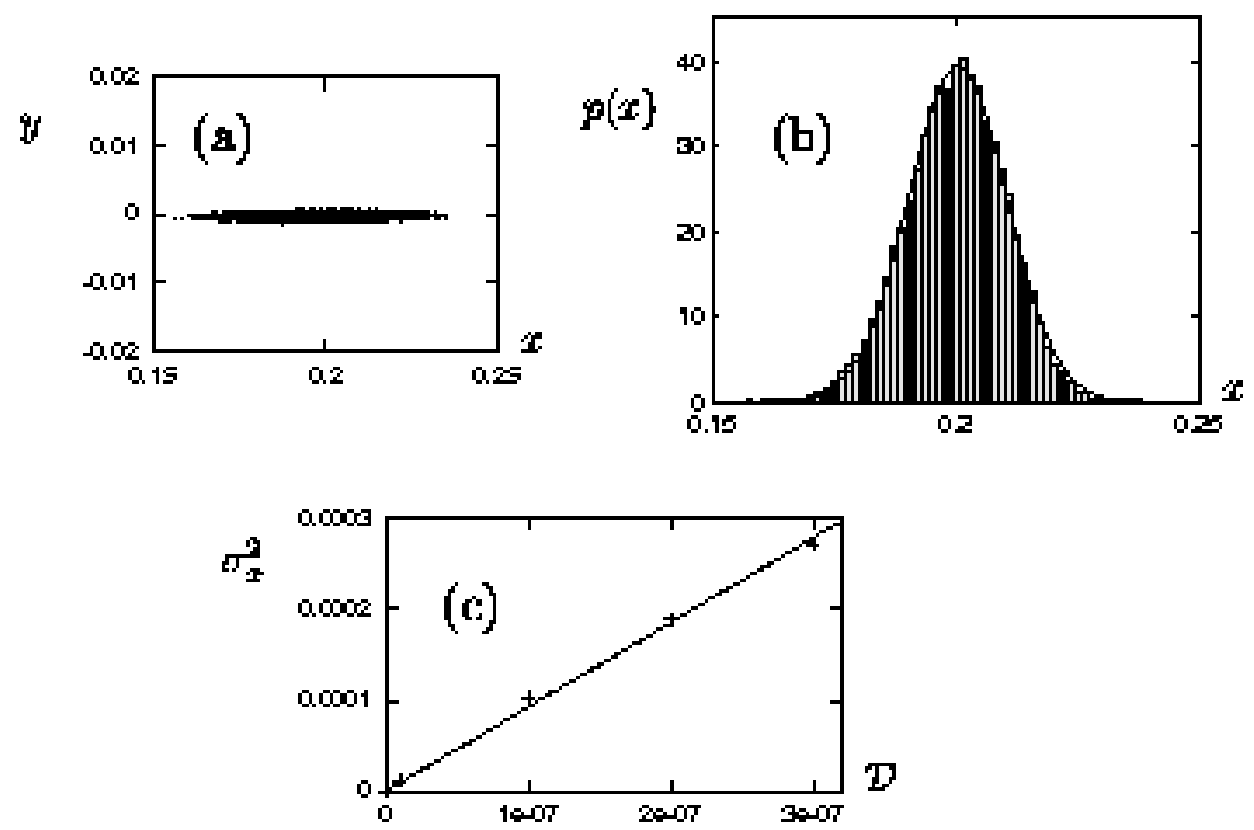

Fig. 2. (a) Values of $(x, y)$ at which the trajectory intersects the surface $\rho=1$ with decreasing $\rho$ for $\mathcal{D}=1 \times 10^{-7}$. The stable manifold of the $u_{\infty}$ solutions intersects the surface $\rho=1$ approximately along the line $x=0$. (b) Probability distribution function $p(x)$ at the surface $\rho=1$. The histogram is from a long-time integration of equations $(1,2)$, and the solid line shows a fit to equation (9). (c) Variance $\sigma_{x}^{2}$ from fitting $p(x)$ to equation (9) for different noise strengths $\mathcal{D}$.

solutions. The probability distribution function (pdf) of $x$ values at the surface $\rho=1$ is shown in Figure 2(b). By definition, $p(x) d x$ is the probability that the $x$ value at the surface $\rho=1$ is between $x$ and $x+d x$. This is fit reasonably well by the Gaussian distribution

$$
p(x)=\frac{1}{\sqrt{2 \pi \sigma_{x}^{2}}} \exp \left[-(x-\bar{x})^{2} /\left(2 \sigma_{x}^{2}\right)\right] .
$$

Figure 2(c) shows that the variances $\sigma_{x}^{2}$ of fitted Gaussians vary linearly with the noise strength $\mathcal{D}$. Over this range $\bar{x} \approx 0.201$, independent of $\mathcal{D}$. The noise strengths have been restricted to the range shown in the figure because for larger $\mathcal{D}$ the noise can kick the trajectory close enough to the stable manifold of a $u_{\infty}$ solution that the long-time integration of equations $(1,2)$ becomes numerically difficult.

We now show how to understand the statistical properties of the bursts in terms of the properties of $p(x)$. In the following, we ignore the strongly contracting $y$ direction. Define $\Sigma_{\rho}=\left\{(x, \rho) \mid \rho=\rho^{*}\right\}$ and $\Sigma_{x}=\left\{(x, \rho) \mid x=x^{*}\right\}$, and consider how the pdf $p(x)$ at $\Sigma_{\rho}$ evolves into a pdf $p(\rho)$ at $\Sigma_{x}$ (see Fig- 


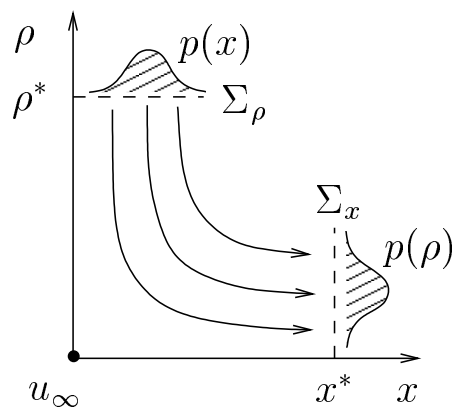

Fig. 3. Setup for determining how the pdf of $x$ values at constant $\rho=\rho^{*}$ evolves into a pdf of $\rho$ values at constant $x=x^{*}$.

ure 3). When the amplitude $r$ becomes large (i.e. when $\rho$ becomes small), the noise terms in equations $(1,2)$ are overwhelmed by the cubic deterministic terms. Therefore, provided $\rho^{*}$ is sufficiently small, we can ignore the noise over the part of the trajectory shown in Figure 3 and approximate the vector field by equation (8). The point $\left(x_{0}, \rho^{*}\right) \in \Sigma_{\rho}$ is mapped into $\left(x^{*}, f\left(x_{0}\right)\right) \in \Sigma_{x}$ under the flow, where $f(x) \equiv \rho^{*}\left(x / x^{*}\right)^{\lambda_{s} / \lambda_{u}}$. Given $p(x)$, the pdf $p(\rho)$ is obtained using $|p(x) d x|=|p(\rho) d \rho|$, with $\rho=f(x)$. For sufficiently small noise we can take $f(x) \approx f(\bar{x})+f^{\prime}(\bar{x})(x-\bar{x})$, where $\bar{x}$ is the average value of $x$ at $\Sigma_{\rho}$. Using (9) as the pdf for $x$ at $\Sigma_{\rho}$, the pdf for $\rho$ at $\Sigma_{x}$ is then

$$
\begin{aligned}
p(\rho) & =\frac{1}{\sqrt{2 \pi \sigma^{2}}} \exp \left[-(\rho-\bar{\rho})^{2} /\left(2 \sigma^{2}\right)\right], \\
\sigma^{2} & =\left(f^{\prime}(\bar{x})\right)^{2} \sigma_{x}^{2}, \quad \bar{\rho}=f(\bar{x}) .
\end{aligned}
$$

In particular, $p(\rho)$ is Gaussian.

Now it is assumed that in the trajectory's successive visits near $u_{\infty}$ solutions, $\rho$ always reaches its minimum value $\rho_{\min }$ at some fixed $x=x^{*}$. This is reasonable because at some $x$ value the trajectory will have been kicked close to a $q p_{\infty}$ solution which is unstable in the $\rho$ direction, so the trajectory starts to evolve towards larger $\rho$ (cf. Figure 1). With this assumption and from (10), the pdf for $\rho_{\min }$ is

$$
p\left(\rho_{\min }\right)=\frac{1}{\sqrt{2 \pi \sigma^{2}}} \exp \left[-\left(\rho_{\min }-\bar{\rho}_{\min }\right)^{2} /\left(2 \sigma^{2}\right)\right]
$$

The pdf for the maximum burst amplitudes, $r_{\max }$, is then obtained using $r_{\max }=1 / \rho_{\min }$ and $\left|p\left(r_{\max }\right) d r_{\max }\right|=\left|p\left(\rho_{\min }\right) d \rho_{\min }\right|:$

$$
p\left(r_{\max }\right)=\frac{1}{r_{\max }^{2} \sqrt{2 \pi \sigma^{2}}} \exp \left[-\left(\frac{1}{r_{\max }}-\bar{\rho}_{\min }\right)^{2} /\left(2 \sigma^{2}\right)\right]
$$



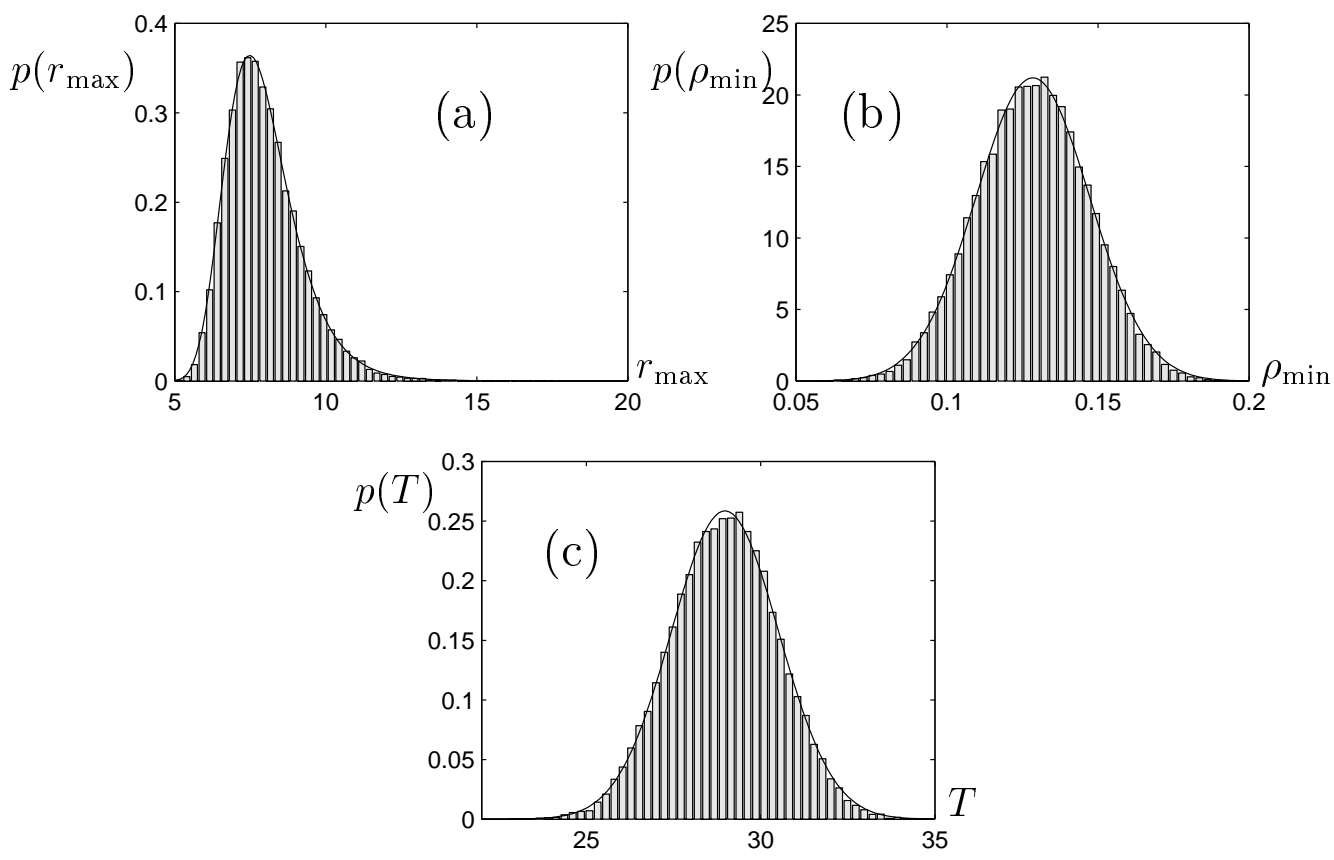

Fig. 4. Probability distribution functions for (a) $r_{\max }$, (b) $\rho_{\min }$, and (c) $T$ for $\lambda=0.1, \mathcal{D}=1 \times 10^{-7}$. The histograms are from a long-time integration of equations $(1,2)$, and the solid lines show fits given by equations (12-14).
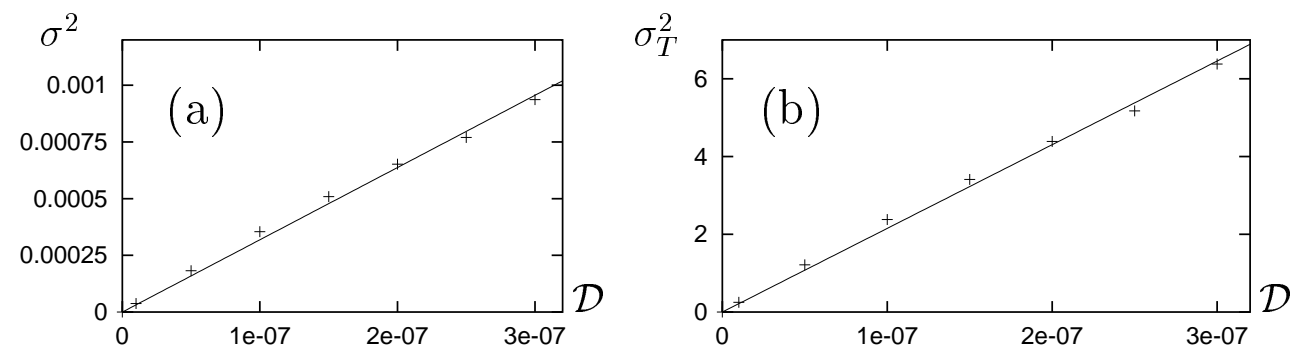

Fig. 5. Linear dependence of the variances (a) $\sigma^{2}$, (b) $\sigma_{T}^{2}$ on the noise strength $\mathcal{D}$.

This distribution has a long $1 / r_{\max }^{2}$ tail for large $r_{\max }$, indicating the probability of bursts having very large amplitudes. These functional forms for the pdf's are verified for $\mathcal{D}=1 \times 10^{-7}$ in Figure $4(\mathrm{a}, \mathrm{b})$. For the range of noise strengths considered, $\bar{\rho}_{\text {min }} \approx 0.128$ independent of $\mathcal{D}$; this is expected from (11) recalling that $\bar{x}$ was found to be independent of $\mathcal{D}$. The variance $\sigma^{2}$ of the fitted distributions is linear in the noise strength $\mathcal{D}$ (see Figure $5(\mathrm{a})$ ). This is also expected from (11) since $\bar{x}$ is independent of and $\sigma_{x}^{2}$ is linear in $\mathcal{D}$.

The statistics for the time $T$ between successive bursts are shown in Figure 4(c) for $\mathcal{D}=1 \times 10^{-7}$. The pdf for $T$ is fit very well by the Gaussian distribution

$$
p(T)=\frac{1}{\sqrt{2 \pi \sigma_{T}^{2}}} \exp \left[-(T-\bar{T})^{2} /\left(2 \sigma_{T}^{2}\right)\right] .
$$


The variance $\sigma_{T}^{2}$ of the fitted distributions is linear in the noise strength $\mathcal{D}$ (see Figure $5(\mathrm{~b})$ ), and $\bar{T} \approx 29.0$ is independent of $\mathcal{D}$. It is interesting to compare these results to the results of [4,5] which considered the effect of noise on attracting, structurally stable heteroclinic cycles connecting finite amplitude fixed points. It was found that the distribution of times between successive visits near the fixed points on the cycle was not Gaussian; instead, it has a long, exponential tail for large times. There is a fundamental reason for the difference between those results and the results presented here: because the heteroclinic cycles considered in $[4,5]$ connect finite amplitude solutions, the trajectory spends most its time in a small neighborhood these solutions. Indeed, this is what allowed a detailed analysis for the times between successive visits near the fixed points to be done. On the other hand, the heteroclinic cycles considered in this paper involve infinite amplitude solutions and are traced out in finite time [8]. More specifically, in the original time $t$ defined by $d t / d \tau=\rho$, equations (8) become

$$
\frac{d x}{d t}=\frac{\lambda_{u} x}{\rho}, \quad \frac{d \rho}{d t}=-\lambda_{s}
$$

Assuming the initial condition $\left(x_{0}, \rho\right) \in \Sigma_{\rho}$ at $t=0$, these equations have the solution $x(t)=x_{0}\left(\rho^{*} /\left(\rho^{*}-\lambda_{s} t\right)\right)^{\lambda_{u} / \lambda_{s}}, \rho(t)=-\lambda_{s} t+\rho^{*}$. The time to go from $\Sigma_{\rho}$ to $\Sigma_{x}$ is thus $t=\left(\rho^{*} / \lambda_{s}\right)\left(1-\left(x_{0} / x^{*}\right)^{\lambda_{s} / \lambda_{u}}\right)$. For the limit $x_{0} \rightarrow 0$ (i.e., as the heteroclinic cycle is approached), $t \rightarrow \rho^{*} / \lambda_{s}$, a finite time. A similar argument shows that the trajectory also returns from infinity in finite time (cf. [8]). This is why the time series in Figure 1(a) shows very rapid growth to and decay from large amplitude. We conclude that the time spent near solutions at infinity is not a dominant part of the total time between bursts. Thus, the results given in $[4,5]$ are not relevant here.

It is not possible to do detailed analytical work for the statistics of the times between bursts because this would require a more detailed understanding of how the trajectory behaves away from the $u_{\infty}$ solutions. However, the following model for the flow between successive bursts gives some qualitative understanding. Consider the formal equation

$$
\frac{d \xi}{d t}=v+\eta(t)
$$

where $v$ is constant, and $\eta$ is a Gaussian white noise random process with $\langle\eta(t)\rangle=0$ and $\left\langle\eta(t) \eta\left(t^{\prime}\right)\right\rangle=2 \mathcal{D} \delta\left(t-t^{\prime}\right)$. If $\xi=\xi_{0}=0$ at $t=0$, the conditional probability distribution function $P\left(\xi, t \mid \xi_{0}\right)$ for solutions of equation (15) obeys the Fokker-Planck equation

$$
\partial_{t} P=-\partial_{\xi}(v P)+\mathcal{D} \partial_{\xi \xi} P, \quad P=\delta(\xi) \text { for } t=0
$$



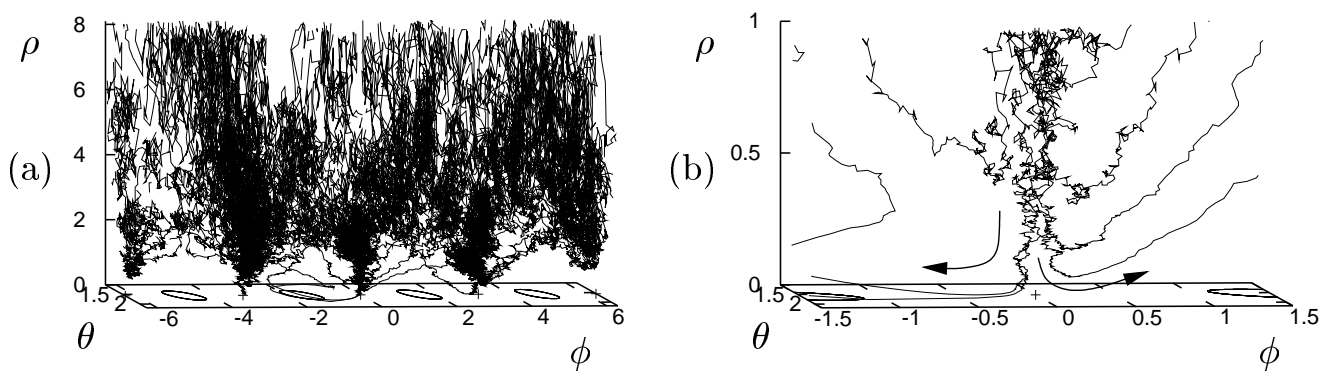

Fig. 6. (a,b) Trajectory for $\lambda=0.1$ and $\mathcal{D}=0.01$. (b) shows the detail near the $u_{\infty}$ solution at $(\rho, \theta, \phi)=(0, \pi / 2,0)$, and the arrows show the sense in which the trajectory is evolving.

This has solution

$$
P\left(\xi, t \mid \xi_{0}=0\right)=\frac{1}{\sqrt{4 \pi \mathcal{D} t}} \exp \left(-(\xi-v t)^{2} /(4 \mathcal{D} t)\right),
$$

i.e., a Gaussian centered at $\xi=v t$ and with variance $2 \mathcal{D} t$. The average time to reach a fixed value $\xi=\xi^{*}$ is then $\bar{T} \equiv \xi^{*} / v$, and the pdf for $\xi$ at this time is $p(\xi) \equiv P\left(\xi, \bar{T} \mid \xi_{0}=0\right)$. We can re-express this in terms of a pdf for the time $T$ at which $\xi=\xi^{*}$ by using the deterministic approximation $T \approx \bar{T}-\left(\xi-\xi^{*}\right) / v$. Then, using $|p(\xi) d \xi|=|p(T) d T|$, we obtain

$$
p(T)=\frac{v}{\sqrt{4 \pi \mathcal{D} \bar{T}}} \exp \left(-v^{2}(T-\bar{T})^{2} /(4 \mathcal{D} \bar{T})\right) .
$$

We make a connection to the distribution of times between successive bursts by thinking of $\xi$ as an arclength coordinate along a trajectory for equations $(1,2)$. Because the trajectory does not spend a dominant part of its time in a neighborhood of the $u_{\infty}$ and $q p_{\infty}$ solutions, equation (15) is a reasonable model for the flow. Assume that the arclength traced out between successive bursts is roughly constant. The qualitative model (15) thus predicts a Gaussian distribution for the time between successive bursts, with the average time between bursts independent of and the variance linear in $\mathcal{D}$. This is precisely what was found numerically.

Figure 6 shows the projection of a numerical solution to equations $(1,2)$ with the same parameter values as for the trajectories shown in Figure 1, except with $\mathcal{D}=0.01$. Unlike the result in Figure $1(\mathrm{~b})$ for much smaller noise strength, here the underlying stable quasiperiodic solution is not even recognizable. The arrows in Figure 6(b) show that the trajectory can make a visit near a $u_{\infty}$ solution and depart either to the "left" or to the "right". In the $(\rho, x, y)$ coordinates introduced above (cf. equation (8)), the trajectory can intersect a surface of constant $\rho$ for either positive or negative $x$ values. Thus, sufficiently large noise can be responsible for the trajectory crossing the stable manifold 

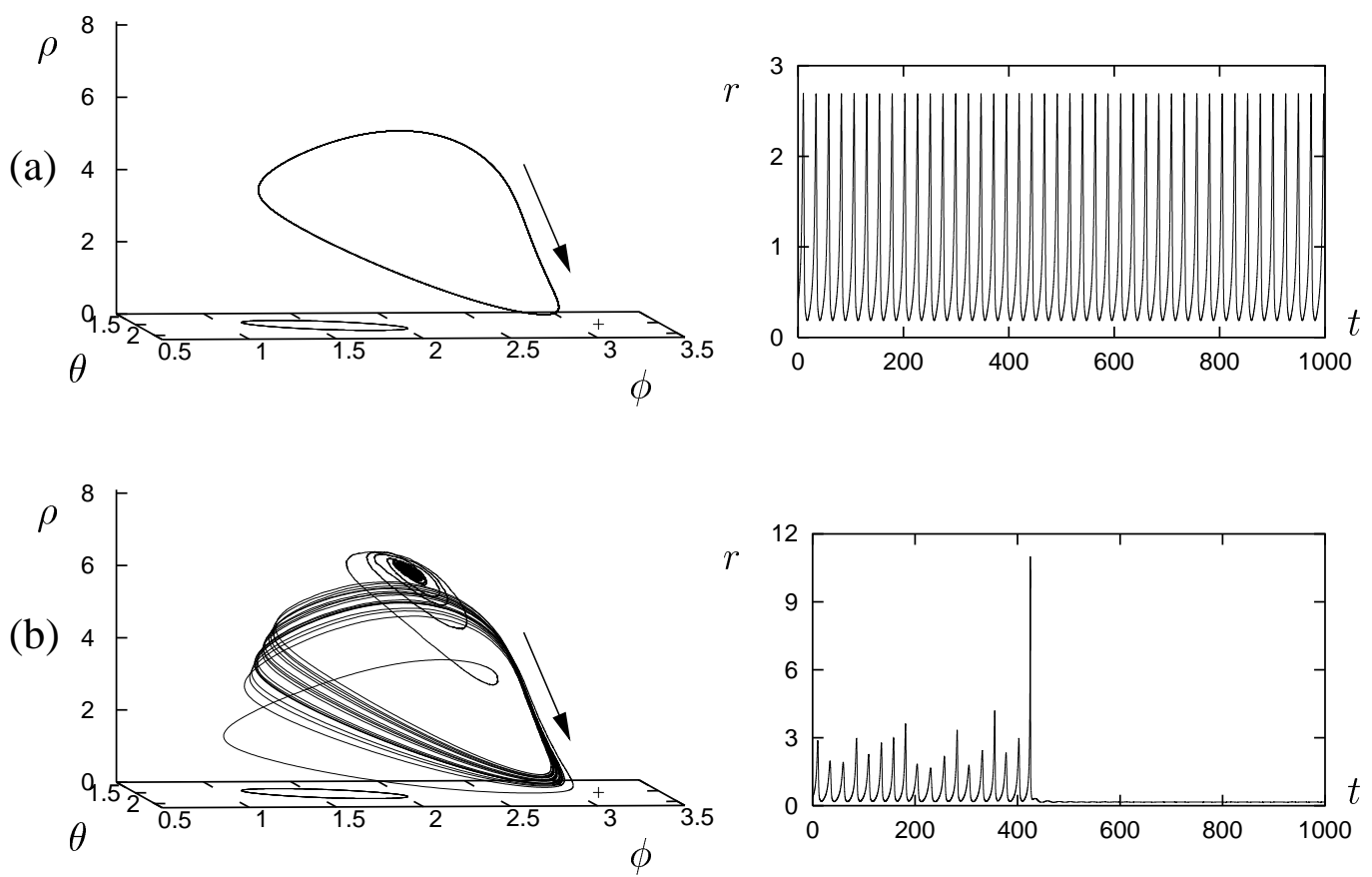

Fig. 7. Bursts for $\lambda=0.1253$ and (a) $\mathcal{D}=0$, (b) $\mathcal{D}=1 \times 10^{-7}$. In (b), it is seen that the noise kicks the system out of the basin of attraction of the state shown in (a) and into the basin of attraction of a solution with constant $\rho=1 / r$.

of the $u_{\infty}$ solutions. This leads to the possibility that successive visits near infinity can either be to different or to the same $u_{\infty}$ solutions. In the convection system, successive visits near the same $u_{\infty}$ solution correspond to successive bursts occurring on the same side of the container [7]. Therefore, sufficiently large noise can affect the physical manifestation of the bursts by destroying purely blinking states.

Reference [7] also identified a different type of stable quasiperiodic solution which is present in the absence of noise for $\lambda=0.1253$ (in the notation of [8], this is a $u / v^{1}$ solution). The trajectory for this solution makes successive visits always near the same $u_{\infty}$ solution (see Figure $7(\mathrm{a})$ ). Physically, this corresponds to a winking state for the convection system because successive bursts occur at the same side of the container [7]. This solution coexists with stable, finite amplitude periodic solutions at $(\rho, \theta, \phi)=(6.298,1.753,2.010 \pm m \pi)$, where $m$ is an integer. Reference [7] argues that these stable, finite amplitude periodic solutions are more likely to be observed in experiments in which the Rayleigh number is ramped upwards, which helps to explain why winking states have apparently never been observed in binary fluid convection experiments. It is seen that even a very small amount of noise $\left(\mathcal{D}=1 \times 10^{-7}\right)$ can kick the system out of the basin of attraction of the winking state and into the basin of attraction of a stable periodic solution (see Figure 7(b)). Thus, even if a winking state could be established in an experiment, it would be 
particularly sensitive to noise.

\section{Noise-induced bursting}

It will now be shown that related bursts may occur for equations $(1,2)$ even in the absence of forced symmetry-breaking, that is, when noise is added to the normal form equations for the Hopf bifurcation with exact $\mathrm{D}_{4}$ symmetry. Specifically, consider equations $(1,2)$ with the same parameters as considered in the previous section $(A=1-1.5 i, B=-2.8+5 i, C=1+i, \omega=1)$, except with $\Delta \lambda=\Delta \omega=0$. For these coefficient values in the absence of noise, a branch of unstable periodic solutions bifurcates subcritically from the trivial state, and two branches of unstable periodic orbits and one branch of globally attracting quasiperiodic solutions all bifurcate supercritically (see [15], also [7,8]). Infinite amplitude periodic and quasiperiodic solutions exist as counterparts of the finite amplitude solutions which bifurcate from the trivial state. In particular, $u_{\infty}$ and $q p_{\infty}$ solutions are the infinite amplitude counterparts of the periodic solutions on the subcritical branch and quasiperiodic solutions on the supercritical branch, respectively [8]. If for some reason the trajectory comes close to the stable manifold of a $u_{\infty}$ solution, it will make an excursion to a neighborhood of that solution, then get kicked toward a $q p_{\infty}$ solution which returns it to smaller amplitude. However, since the finite amplitude quasiperiodic solutions are globally attracting, heteroclinic cycles involving infinite amplitude solutions cannot form, so such a burst is a transient phenomenon. A stable quasiperiodic solution is shown for $\lambda=0.1, \mathcal{D}=0$ in Figure 8(a). For small noise the trajectory remains near the quasiperiodic solution (see Figure $8(\mathrm{~b})$ ). However, sufficiently large noise can repeatedly kick the system close to the stable manifold of a $u_{\infty}$ solution, leading to bursts of very large dynamic range (see Figure $8(\mathrm{c})$ ). This is highly reminiscent of results for the effect of resonant temporal forcing on the Hopf bifurcation with $\mathrm{D}_{4}$ symmetry [13]; there it was found that as the forcing amplitude increased, the attractor came closer to the stable manifolds of $u_{\infty}$ solutions, leading to bursting behavior.

As a further demonstration of the effect of noise on a Hopf bifurcation with exact $\mathrm{D}_{4}$ symmetry, we close by considering an attracting infinite amplitude heteroclinic cycle. Specifically, consider the parameters $A=0.1-1.5 i, B=$ $-1-4 i, C=1-2 i, \lambda=0.1, \omega=1, \Delta \lambda=\Delta \omega=0$. Using the results of [15], it can be shown that there are heteroclinic connections between $u_{\infty}$ solutions within the $\Sigma$ subspace. Linearizing equations (4-6) about the $u_{\infty}$ solutions shows that the eigenvalue corresponding to perturbations in the $\rho$ direction is -0.2 , while the eigenvalues corresponding to perturbations within the $\Sigma$ subspace are -4.828 and 0.828 . Since the first eigenvalue is negative and the sum of the latter two eigenvalues is also negative, the heteroclinic cycle con- 

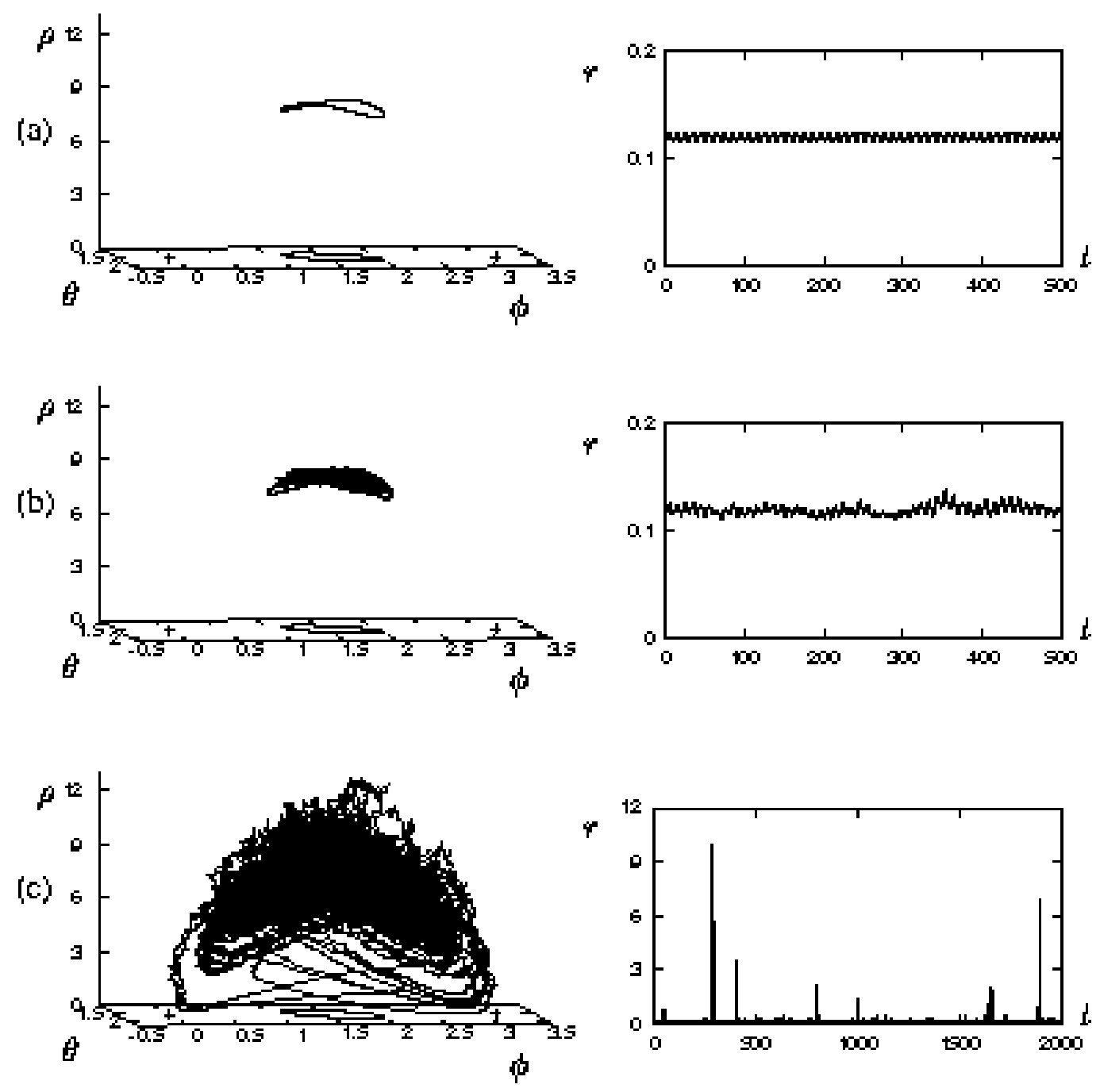

Fig. 8. Solutions for $\lambda=0.1$ and (a) $\mathcal{D}=0$, (b) $\mathcal{D}=1 \times 10^{-6}$, (c) $\mathcal{D}=1 \times 10^{-4}$ with $\Delta \lambda=\Delta \omega=0$. Note the different scales for the time series plots.

necting $u_{\infty}$ is attracting (cf. [15]). Figure 9 shows a numerically calculated trajectory for these parameter values and $\mathcal{D}=1 \times 10^{-5}$; the time step of integration is $\delta t=1 \times 10^{-6}$. Much like the situations described in [3-5], noise causes the attracting heteroclinic cycle to become a "statistical limit cycle" in which switching between $u_{\infty}$ solutions occurs randomly in time but with a well-defined mean period. 


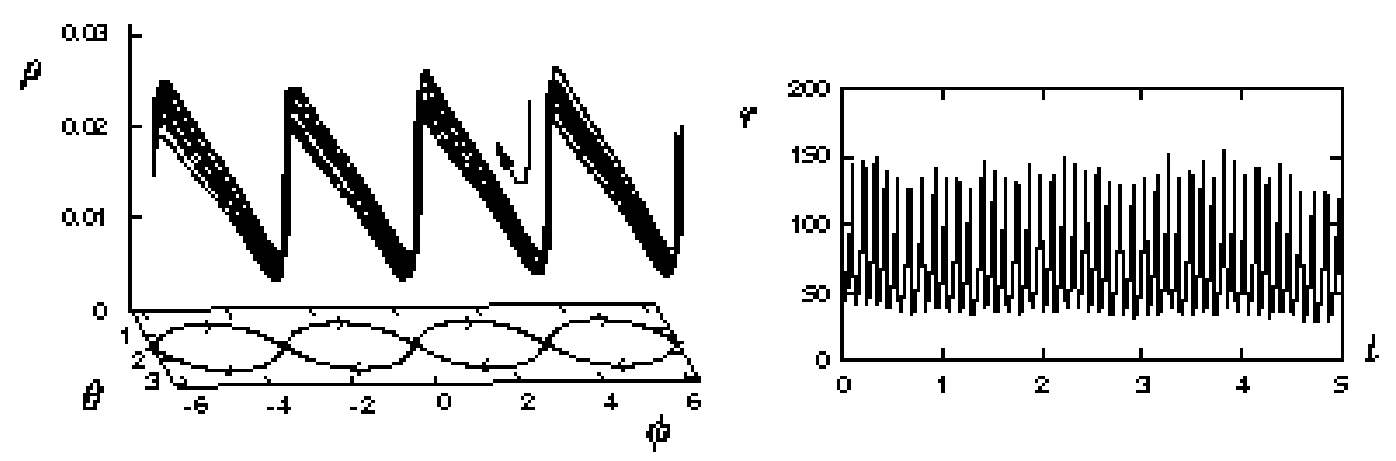

Fig. 9. The left figure shows the attracting infinite amplitude heteroclinic cycle connecting $u_{\infty}$ solutions at $\rho=0$, and the "statistical limit cycle" solution for $\mathcal{D}=1 \times 10^{-5}$. The right figure shows that successive visits near $u_{\infty}$ solutions occurs randomly, but with a well-defined mean period.

\section{Conclusion}

The effect of additive white noise on a model for large aspect-ratio binary fluid convection has been considered. Particular attention has been paid to the effect of noise on bursting behavior present in the model. It was shown that even a very small amount of noise can have a very large effect on the amplitudes of successive bursts. This is because there will be a burst with larger amplitude if the noise kicks the system closer to the stable manifold of an infinite amplitude state, and a burst with smaller amplitude if the noise kicks it away. Numerical results and analytical arguments were given to understand the statistical properties of bursts in the presence of noise. It was also demonstrated that large enough noise can affect the physical manifestations of the bursts by destroying purely blinking and purely winking states. In fact, winking states are particularly sensitive to noise, which helps to explain why they have apparently never been observed in experiments. Finally, it was shown that related bursts can occur when noise is added to the normal form equations for the Hopf bifurcation with exact square symmetry.

Reasoning similar to that given in [8] suggests that this type of bursting behavior can persist when higher terms in equations $(1,2)$ are retained, even in the presence of noise. Bursts will then be associated with visits near large but finite amplitude solutions. This is important because it implies that bursts similar to those described in this paper may be observed in real physical systems undergoing a Hopf bifurcation with exact or weakly broken $\mathrm{D}_{4}$ symmetry; the (unphysical) infinite amplitude solutions are not necessary. There is also a possibility that in appropriate cases the noise could be more important than higher order terms so their exact nature would be irrelevant.

Other physical systems for which the results presented in the paper might be 
relevant because their evolution equations are related to the Hopf bifurcation with $\mathrm{D}_{4}$ symmetry include any system in a square domain undergoing an oscillatory instability [17] or displaying oscillatory patterns which are periodic on a square lattice [18], electrohydrodynamic convection in liquid crystals [19], lasers [20], spring-supported fluid-conveying tubes [21], the Faraday system in a square or nearly-square container [22], and dynamo theories of magnetic field generation in the Sun $[10,23]$.

Acknowledgements: This work was supported by a National Science Foundation Mathematical Sciences Postdoctoral Research Fellowship. I would like to thank Edgar Knobloch and Philip Holmes for useful comments and discussions.

\section{References}

[1] G. D. Lythe and M. R. E. Proctor, Noise and slow-fast dynamics in a three-wave resonance problem, Phys. Rev. E 47 (1993) 3122-3127.

[2] E. Stone and D. Armbruster, Noise and $\mathrm{O}(1)$ amplitude effects on heteroclinic cycles, Chaos 9 (1999) 499-506.

[3] F. H. Busse and K. E. Heikes, Convection in a rotating layer: a simple case of turbulence, Science 208 (1980) 173-175.

[4] E. Stone and P. Holmes, Noise induced intermittency in a model of a turbulent boundary layer, Physica D 37 (1989) 20-32.

[5] E. Stone and P. Holmes, Random perturbations of heteroclinic attractors, SIAM J. Appl. Math 50 (1990) 726-743.

[6] A. S. Landsberg and E. Knobloch, Oscillatory bifurcation with broken translation symmetry, Phys. Rev. E 53 (1996) 3579-3600.

[7] J. Moehlis and E. Knobloch, Forced symmetry breaking as a mechanism for bursting, Phys. Rev. Lett. 80 (1998) 5329-5332.

[8] J. Moehlis and E. Knobloch, Bursts in oscillatory systems with broken $\mathrm{D}_{4}$ symmetry, Physica D 135 (2000) 263-304.

[9] T. S. Sullivan and G. Ahlers, Nonperiodic time dependence at the onset of convection in a binary liquid mixture, Phys. Rev. A 38 (1988) 3143-3146.

[10] E. Knobloch and A. S. Landsberg, A new model of the solar cycle. Mon. Not. R. Astr. Soc. 278 (1996) 294-302.

[11] E. Knobloch and J. Moehlis, Bursting mechanisms for hydrodynamical systems, in Pattern Formation in Continuous and Coupled Systems: A Survey Volume, ed. M. Golubitsky, D. Luss, and S. H. Strogatz, Springer-Verlag, New York, (1999) 157-174. 
[12] E. Knobloch and J. Moehlis, Burst mechanisms in hydrodynamics, in Nonlinear Instability, Chaos, and Turbulence, Vol. II, ed. L. Debnath and D. Riahi, Computational Mechanics Publications, Southampton, (2000) 237-287.

[13] J. Moehlis and E. Knobloch, Wrinkled tori and bursts due to resonant temporal forcing, to appear in Physica D.

[14] O. Batiste, I. Mercader, M. Net, and E. Knobloch, Onset of oscillatory binary fluid convection in finite containers, Phys. Rev. E 59 (1999) 6730-6741.

[15] J. W. Swift, Hopf bifurcation with the symmetry of the square, Nonlinearity 1 (1988) 333-377.

[16] R. L. Honeycutt, Stochastic Runge-Kutta algorithms. I. White noise, Phys. Rev. A 45 (1992) 600-603.

[17] P. Ashwin and Z. Mei, Normal form for Hopf bifurcation of partial differential equations on the square, Nonlinearity 8 (1995) 715-734.

[18] M. Silber and E. Knobloch, Hopf bifurcation on a square lattice, Nonlinearity 4 (1991) 1063-1107.

[19] H. Riecke, M. Silber, and L. Kramer, Temporal forcing of small-amplitude waves in anisotropic systems, Phys. Rev. E 49 (1994) 4100-4113.

[20] Q. Feng, J. V. Moloney and A. C. Newell, Transverse patterns in lasers, Phys. Rev. A 50 (1994) R3601-3604.

[21] A. Steindl and H. Troger. One and two-parameter bifurcations to divergence and flutter in the three-dimensional motions of a fluid conveying viscoelastic tube with $\mathrm{D}_{4}$ symmetry, Nonlinear Dynam. 8 (1995) 161-178.

[22] F. Simonelli and J. P. Gollub, Surface wave mode interactions: effects of symmetry and degeneracy, J. Fluid Mech. 199 (1989) 471-494.

[23] E. Knobloch, S. M. Tobias and N. O. Weiss, Modulation and symmetry changes in stellar dynamos, Mon. Not. R. Astr. Soc. 297 (1998) 1123-1138. 